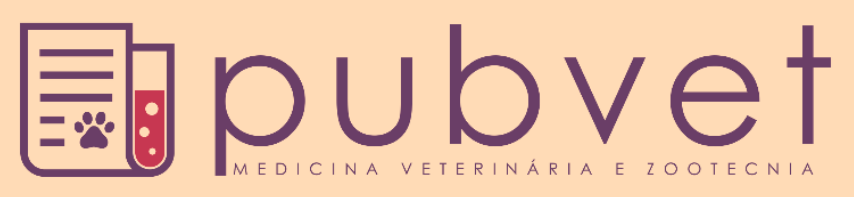

https://doi.org/10.31533/pubvet.v15n04a797.1-7

\title{
Difilobotríase: considerações sobre uma doença emergente
}

\author{
Laura Costa Borges $^{1 * \bullet}$, Ligia Cantarino ${ }^{2}$, Simone Perecmanis $^{20}$ \\ ${ }^{1}$ Médica-Veterinária autônoma. \\ ${ }^{2}$ Docentes da Faculdade de Agronomia e Medicina Veterinária da Universidade de Brasília. Brasília-DF. \\ *Autor para correspondência, E-mail: lcostaborges@gmail.com
}

\begin{abstract}
Resumo A difilobotríase ou difilobotriose é uma zoonose causada pelo cestódeo Diphyllobothrium spp. pelo consumo de peixes infectados crus ou mal cozidos. É uma das mais importantes doenças transmitidas por pescados, sendo amplamente distribuída pelo mundo. Mamíferos (incluindo os humanos) e aves piscívoras são hospedeiros definitivos do parasito, que possui um ciclo complexo que incluí ao menos dois hospedeiros intermediários como crustáceos, peixes, aves e mamíferos. Diversas espécies conhecidas do Diphyllobothrium spp. podem infectar o homem, podendo ser diferenciadas e identificadas por métodos de biologia molecular. O cozimento adequado do pescado e o congelamento prévio são suficientes para eliminar o parasita dos alimentos.
\end{abstract}

Palavras chave: difilobotríase, pescados, zoonose

\section{Diphyllobothriasis: considerations on an emerging disease}

\begin{abstract}
Diphyllobothriasis is a zoonoses caused by Diphyllobothrium spp. cestode by consumption of infected raw or undercooked fish. Is one of the most important diseases transmitted by fish and widely distributed around the world. Mammals (including humans) and piscivorous birds are permanent parasitic hosts, which have a complex cycle that include at least two intermediate hosts, such as crustaceans, fishes, birds and mammals. Some Diphyllobothrium spp. species are known to infect humans and they can be differentiated and identified by molecular biology methods. Properly cooking the fish and deep-freezing it are sufficient to eliminate the parasite in food.
\end{abstract}

Keywords: Diphyllobothriasis, fish, zoonosis

\section{Introdução}

O Regulamento de Inspeção Industrial e Sanitária de Produtos de Origem Animal (RIISPOA) do Ministério da Agricultura, Pecuária e Abastecimento (MAPA), em seu artigo 205, define que pescado compreende peixes, crustáceos, moluscos, anfíbios, répteis, equinodermos e outros animais aquáticos usados na alimentação humana (Brasil, 2017).

O pescado constitui importante fonte de proteína animal para a alimentação humana por ser rico em aminoácidos essenciais. A Organização Mundial da Saúde (OMS) recomenda que o consumo de pescados deva ser de $12 \mathrm{~kg} / \mathrm{hab}$./ano (Sartori \& Amancio, 2012). O consumo mundial de pescado tem aumentado, segundo a Organização das Nações Unidas para Alimentação e Agricultura (FAO). A produção mundial de pescados em 2009 foi de 145 milhões de toneladas (Sonoda \& Shirota, 2012) e em 2016 foi superior a 170 milhões de toneladas (FAO, 2018). No Brasil, a produção da aquicultura e pesca foi superior a 1,2 milhões de toneladas em 2016, o consumo per capita de peixe apresentou aumento de 4,6 kg/hab./ano em 1961 para 9,7 kg/hab./ano em 2013, ainda abaixo do recomendado pela OMS (FAO, 2018).

O Brasil é um importador de pescados, embora parte dos brasileiros não tenha o hábito de consumilos. Entre as famílias que consomem pescado, o consumo médio passa de 27,2 kg/hab/ano. Existe 
consumo diferenciado entre as regiões brasileiras: Norte e Nordeste são responsáveis por quase $70 \%$ do consumo domiciliar de pescado e a região Centro-Oeste é responsável por 3\% (López-López et al., 2009; Sonoda \& Shirota, 2012).

O aumento do consumo de pescado deve ser acompanhado por ações de vigilância, pois pode acarretar aumento de zoonoses parasitárias. Zoonose é definida por qualquer doença ou infecção naturalmente transmissível de animais vertebrados para seres humanos, podendo ser causada por um vírus, bactéria ou parasito (OMS, 2016). No Brasil, foram identificados os agentes de 2350 surtos de doenças veiculadas por alimentos de 2009 a 2018, sendo pelo menos $28,4 \%$ de produtos de origem animal e, destes, $2,1 \%$ relacionados ao consumo de pescados, frutos do mar e seus derivados (Brasil, 2019).

A tendência global para o aumento do consumo de pescados pode estar associada a uma linha saudável de alimentação; com o aumento do número mundial de viagens nacionais e internacionais, $o$ que favorece a intercâmbios culturais incluindo alteração de hábitos alimentares como a popularização da culinária asiática, principalmente a japonesa (Tavares et al., 2018).

A difilobotríase ou difilobotriose, helmintose zoonótica era mais descrita em regiões frias. No entanto, com a globalização a doença passou a ser amplamente distribuída. A doença é uma das mais antigas conhecidas pelo homem, sendo relatada desde a antiguidade. Atualmente é considerada doença emergente e reemergente com aumento do diagnóstico em vários países como Rússia, Coreia do Sul, Japão e na América do Sul, com casos registrados no Brasil (Kuchta et al., 2014; Scholz \& Kuchta, 2016). No Brasil, têm sido relatados casos em diversas cidades que têm como causa o consumo de peixe cru proveniente de criações de salmão do sul do Chile (Cabello, 2007).

\section{Etiologia}

O Diphyllobothrium spp., ou a "tênia do peixe", como é conhecida, é um verme platelminto da classe Cestoda, de ordem Pseudophyllidea, da família Diphyllobothriidea e gênero Diphyllobothrium (Machado \& Marques, 2015). É um parasito longo, que pode chegar a $20 \mathrm{~m}$ de comprimento, sendo o maior cestódeo a parasitar o homem e originar a difilobotríase, uma das mais importantes doenças transmitidas por peixes (Rosas \& Weitzel, 2014).

\section{Morfologia}

Os vermes adultos são compostos por três regiões: escólex, pescoço e proglotes. No escólex (cabeça) fica localizado um par de órgãos de fixação, os bothrios, sulcos musculares alongados ventrais e dorsais que fornecem fixação por meio da compressão do tecido do hospedeiro entre eles, como ventosas (Cimerman \& Cimerman, 2005; Mega et al., 2013; Neves, 2005). O pescoço é a região intermediária, onde ocorre a proliferação de novas proglotes, podendo conter até 5.000 proglotes formando seu corpo, também chamado de estróbilo (Mega et al., 2013; Rosas \& Weitzel, 2014). As proglotes são caracterizadas pelo hermafroditismo como na maioria dos cestódeos, possuindo pelo menos um par de órgãos sexuais, masculino e feminino, os testículos e poros genitais. Na parte dorsal das proglotes encontram-se cerca de 700 a 800 testículos, já na face ventral, encontram-se dois poros genitais: o anterior é composto pela vagina e canal deferente e o posterior é o tocóstomo, orifício de postura (Machado \& Marques, 2015). Em cada proglote, pode ser visualizado um útero tubular, que pode gerar vários ovos, e diversos folículos na camada cortical que irão fornecer o material genético (Kuchta et al., 2014; Scholz \& Kuchta, 2016).

A característica marcante nas proglotes grávidas é a presença de ovos escuros (Kuchta et al., 2014; Scholz \& Kuchta, 2016). Os ovos são ovais, com um opérculo em uma extremidade e corpúsculo polar no lado oposto (Rosas \& Weitzel, 2014; Santos \& Faro, 2005) (Figura 1). Eles não contêm embrião completamente desenvolvido, já que ele completa seu desenvolvimento após a expulsão dos ovos de proglotes grávidas na água (Kuchta et al., 2014; Scholz \& Kuchta, 2016).

\section{Primeiro hospedeiro intermediário}

Os coracídios circulam pela água e são ingeridos por micro crustáceos planctônicos (normalmente copépodes), sendo diversas espécies de crustáceos passíveis de serem os primeiros hospedeiros intermediários. Já dentro do novo hospedeiro, perdem seus cílios e penetram pela parede intestinal, onde pegam nutrientes e se desenvolvem, tornando-se larvas procercóide (Santos \& Faro, 2005). 


\section{Segundo hospedeiro intermediário}

As larvas procercóide continuam em seus hospedeiros até que eles sejam ingeridos por um segundo hospedeiro intermediário, normalmente um peixe como o salmão que possui grande predileção por esses crustáceos. Após infectarem o segundo hospedeiro, o parasito penetra pelo intestino, perde seu cercômero e se instala na musculatura onde se torna uma plerocercóide. Se acontecer de o segundo hospedeiro intermediário ser ingerido por um peixe predador ainda maior, as plerocercóides migram para a musculatura do novo peixe. As larvas plerocercóides possuem poucos centímetros de comprimento, cor esbranquiçada, normalmente se alojam dentro da musculatura e podem formar

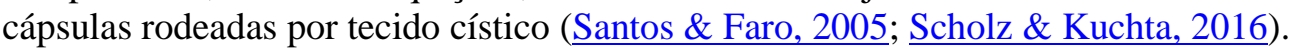

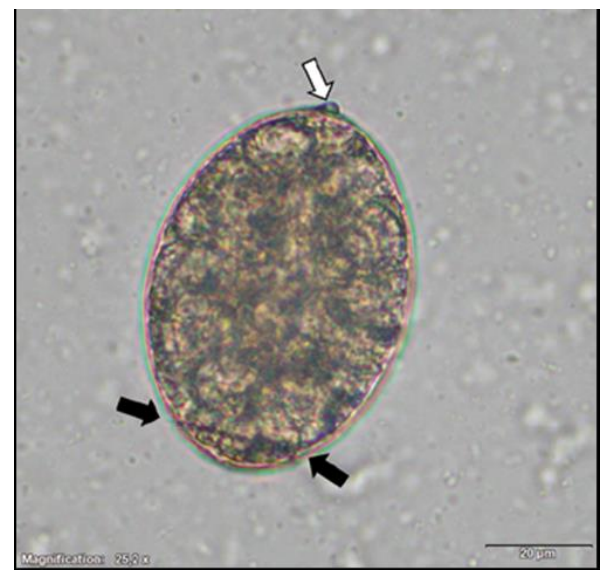

Figura 1. Ovo de Diphyllobothrium latum. As setas pretas indicam a região do corpúsculo polar e a seta branca indica o opérculo. Fonte: Rosas \& Weitzel (2014).

\section{Hospedeiro definitivo}

Quando as larvas plerocercóides são ingeridas pelo hospedeiro definitivo, elas passam pelo estômago, o escólex se firma na mucosa intestinal e se desenvolvem rapidamente, produzindo ovos

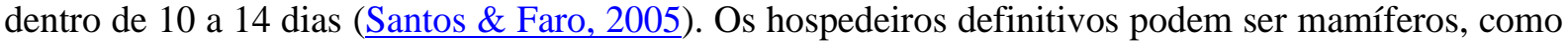
o homem, cães, lobos, raposas, focas e ursos, além de algumas aves pesqueiras (Brasil, 2007; Scholz \& Kuchta, 2016) (igura 2).

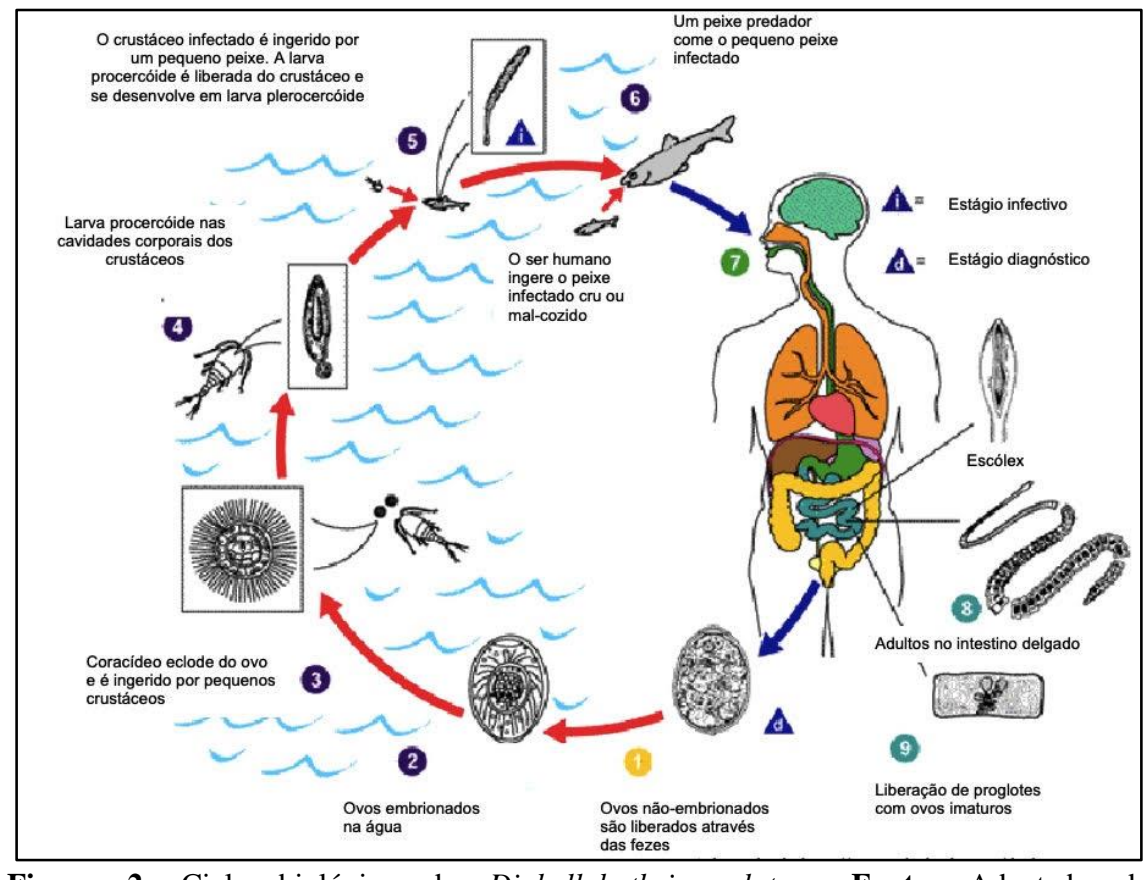

Figura 2. Ciclo biológico do Diphyllobothrium latum. Fonte: Adaptado de http://www.dpd.cdc.gov/dpdx por Brasil (2007). 


\section{Epidemiologia}

A infecção por Diphyllobothrium spp. no homem se dá pela ingestão de pescados frescos contaminados crus, mal cozidos, defumados ou marinados. Consequentemente, acometem mais as populações perto de mares, rios, lagos e com longa tradição de consumo de pescados, principalmente nas regiões de águas mais frias onde podemos encontrar determinados tipos de peixes (Kuchta et al., 2014; Scholz \& Kuchta, 2016).

Os peixes mais frequentemente infectados pelo parasito são os frescos de água doce ou de água salgada que se reproduzem em água doce, como salmão e trutas. Outros peixes como a perca (Perca fluviatilis) e o lúcio (Esox lucius) também podem se infectar (Santos \& Faro, 2005; Scholz \& Kuchta, 2016). Na América do Sul, salmonídeos selvagens e de criatórios como a truta arco-íris (Oncorhynchus mykiss) foram reportados como hospedeiros intermediários (Scholz \& Kuchta, 2016).

De acordo com Scholz \& Kuchta (2016), várias espécies do Diphyllobothrium são capazes de infectar o homem. Das 15 conhecidas, 4 são mais relatadas: Diphyllobothrium latum, Diphyllobothrium nihonkaiense, Diphyllobothrium dendriticum e Adenocephalus pacificus. O D. latum ocorre mais na América do Norte e na região da Euro-Sibéria, e é o mais frequentemente encontrado em casos humanos no mundo. O D. nihonkaiense, o segundo mais comum, ocorre na costa do Pacífico norte na Ásia e na zona oeste da América do Norte. O D. dendriticum é relatado na região do Holoártico, sendo mais propagado que o $D$. latum por possuir pássaros como hospedeiros definitivos, e também é encontrado na região da Patagônia. Já o A. pacificus, ou D. pacificum como também é conhecido, é mais comumente encontrado na Costa Pacífica da América do Sul, apesar de haver relatos na Espanha, Japão e Rússia.

\section{Diagnóstico, prevenção e tratamento}

A maioria das infecções por Diphyllobothrium é assintomática. Mas, quando presentes, os sinais clínicos são compatíveis com os de outras parasitoses intestinais, como: dores abdominais, diarreia recorrente, vômitos e perda de peso. O parasito compete com o hospedeiro por vitamina B12, podendo levar à sua deficiência e, consequentemente, anemia megaloblástica. Constipação, fadiga, dores de cabeça e reações alérgicas também podem ocorrer (Brasil, 2009; Emmel et al., 2006; Mega et al., 2013). Obstrução do intestino, inflamação do ducto ou vesícula biliar por migração de proglotes são algumas das complicações descritas (Mega et al., 2013; OMS, 1995).

O diagnóstico em humanos se baseia na presença de ovos operculados (Marval et al., 2013) e pedaços do estróbilo em amostras de fezes. A colonoscopia, um importante forma diagnóstica, também pode ser um método para identificar a presença do verme adulto, como evidenciado por Park et al. (2011) acidentalmente em um exame.

O formato das proglotes e a posição média dos poros genitais fazem com que sua diferenciação de outros platelmintos seja fácil, porém a sua diferenciação por espécie pode ser difícil sem a presença do escólex, já que este quase nunca é liberado nas fezes (Mega et al., 2013). Portanto, a única maneira confiável de identificação da espécie infectante se dá por uso de técnicas moleculares, especialmente pela sequência genética cox 1 (Scholz \& Kuchta, 2016).

A inspeção dos pescados é fundamental para a identificação e controle de parasitos nas indústrias. Nos estabelecimentos de pescados, a utilização da mesa de luz branca é uma maneira de identificar o parasito na musculatura do pescado por inspeção visual do mesmo. O método se dá pela colocação dos filés descongelados em uma superfície acrílica de aproximadamente $5 \mathrm{~mm}$ (cinco milímetros) de espessura e $45 \%$ de transparência com a incidência de luz branca por baixo da placa de aproximadamente 1500 lux. Pela incidência de luz é possível identificar a presença do parasito, que pode acarretar perdas devido ao aspecto repugnante causado no pescado, levando à rejeição pelo consumidor ou condenação total por parte do estabelecimento (Brasil, 2018).

A difilobotríase pode ser prevenida pela ingestão de peixes bem cozidos ou que passaram pelo processo de congelamento prévio. A Food and Drug Administration (FDA, 2011) e a Agência Nacional de Vigilância Sanitária (Anvisa) (Brasil, 2007) recomendam que o peixe passe por processo de congelamento a $-35^{\circ} \mathrm{C}$ por 15 horas após atingir o estado sólido, $-20^{\circ} \mathrm{C}$ por 24 horas após atingir o estado sólido ou em congelador convencional com temperatura até $-20^{\circ} \mathrm{C}$ por 7 dias. Este processo de 
congelamento é eficaz não somente para matar o Diphyllobothrium spp., mas também outros parasitos de peixes que podem causar problemas de saúde (FDA, 2011).

No artigo 216 do RIISPOA (Brasil, 2017) não há regulamentação de temperaturas de tratamento de pescados por congelamento até $-20^{\circ} \mathrm{C}$, apenas a $-20^{\circ} \mathrm{C}$ e $-35^{\circ} \mathrm{C}$ que o consumidor normalmente não tem acesso. Todavia, o referido artigo deixa em aberto a possibilidade de serem utilizados processos diferentes dos propostos desde que embasados técnico-cientificamente e aprovados pelo Departamento de Inspeção de Produtos de Origem Animal (DIPOA).

O tratamento da doença em humanos se dá pela utilização do anti-helmíntico Praziquantel em dose única de $10-25 \mathrm{mg} / \mathrm{kg}$ que age em larvas e vermes adultos. A maioria das larvas são eliminadas mesmo quando incrustadas e se desintegram por completo dentro de 5 meses. Também é possível utilizar Niclosamida em dose única de $2 \mathrm{~g}$ para adultos, $1 \mathrm{~g}$ para crianças de $10 \mathrm{~kg}$ a $35 \mathrm{~kg}$ e $0,5 \mathrm{~g}$ para crianças com menos de 10 kg (OMS, 1995; Brasil, 2010). Em pacientes com infestação crônica, a administração deste pode ser feita em associação com laxante para aumentar a eficácia do tratamento. $\mathrm{O}$ antiparasitário atua no bloqueio da captação de glicose pelos vermes intestinais (

O tratamento nos pescados é muito mais complexo em virtude da água estar contaminada, sendo lagoas, rios e mares áreas de difícil controle, tornando-se um problema de importância para a saúde pública. Por este fato o tratamento do esgoto é fundamental para eliminar os ovos das fezes humanas e impedir a contaminação de micro crustáceos presentes na água (Machado \& Marques, 2015). Já em áreas endêmicas, devem-se eliminar os micros crustáceos, hospedeiros intermediários, para evitar a presença dos parasitos na criação de peixes (Luque, 2004).

\section{Considerações finais}

A difilobotríase ou difilobotriose é uma doença emergente de distribuição global e afeta aqueles que se alimentam de peixes contaminados crus, mal cozidos, marinados e defumados. O perfil epidemiológico da doença e das demais doenças transmitidas por alimentos tem apresentado mudanças, esse fato pode ocorrer pela introdução de novos hábitos alimentares, aumento de viagens internacionais migração humana pelo turismo, as melhorias nos sistemas de transporte e o crescimento do mercado internacional de alimentos - Globalização.

É de grande importância que a população e os trabalhadores da área devam ser alertados para esse agravo: alimentos como pescados devem ter origem conhecida, acondicionamento e armazenamento adequados, assim como tratamento térmico conforme as orientações das autoridades sanitárias.

As principais medidas preventivas e de controle estão centradas em prevenção da contaminação da água; no tratamento das pessoas acometidas pelo parasito e na prevenção da transmissão de larvas infectantes dos peixes aos humanos. Saneamento básico, estações de tratamento de esgoto, medidas de higiene e educação em saúde são fundamentais para esse processo.

Mais estudos devem ser feitos para melhor entendimento da doença, além de chamar atenção da população em geral para esse problema de Saúde Pública.

\section{Referências}

BRASIL. Agência Nacional de Vigilância Sanitária/ Secretaria de Vigilância à saúde/ Ministério da Saúde/ Ministério da Agricultura, Pecuária e Abastecimento. Difilobotríase: Comunicado ANVISA/SVS/MS/MAPA. Alerta e recomendações referentes a casos de Difilobotríase no município de São Paulo 2007. Disponível em: <http://www.saude.sp.gov.br/resources/cve-centrode-vigilancia-epidemiologica/areas-de-vigilancia/doencas-transmitidas-por-agua-ealimentos/doc/2005/com4_alertams.pdf>. Acesso em: 15 fev. 2019.

BRASIL. Centro de Vigilância Epidemiológica. CVE. Divisão de Doenças de Transmissão Hídrica e Alimentar, Secretaria de Estado da Saúde de São Paulo. Difilobotríase: casos autóctones da tênia do peixe (D. latum) identificados no município de São Paulo e outras cidades do Estado de São Paulo. 2009. Disponível em: <http://www.saude.sp.gov.br/resources/cve-centro-de-vigilanciaepidemiologica/areas-de-vigilancia/doencas-transmitidas-por-agua-e-

alimentos/doc/2009/2009comunicado_medicos_laboratorios.pdf>. Acesso em 17 fev. 2019. 
BRASIL. Ministério da Saúde. Secretaria de Vigilância em Saúde. Departamento de Vigilância Epidemiológica. Manual integrado de vigilância, prevenção e controle de doenças transmitidas por alimentos. Ministério da Saúde, Secretaria de Vigilância em Saúde, Departamento de Vigilância Epidemiológica. - Brasília: Editora do Ministério da Saúde, 2010. 158 p.: il. - (Série A. Normas e Manuais Técnicos).

BRASIL. (2017). Ministério da Agricultura, Pecuária e Abastecimento. Regulamento da Inspeção Industrial e Sanitária de Produtos de Origem Animal - RIISPOA. Brasília.

BRASIL. (2018). Ministério da Agricultura, Pecuária e Abastecimento. Memorando-Circular $\mathrm{n}^{\circ}$ 2/2018/CGI/DIPOA/MAPA/SDA/MAPA, 2018. Disponível em: <http://seafoodbrasil.com.br/wpcontent/uploads/2018/02/Memorando-Circular-CGI-02-18.-Controle-oficail-de-parasitas-empescado.-SEI_21000.004629_2018_36.pdf> Acesso em 25 Fev. 2019.

BRASIL. Ministério da Saúde. Secretaria de Vigilância em Saúde. Departamento de Vigilância das Doenças Transmissíveis. Surtos de Doenças Transmitidas por Alimentos no Brasil: Informe 2018. 2019. Disponível em: http://portalarquivos2.saude.gov.br/images/pdf/2019/fevereiro/15/Apresenta----o-Surtos-DTA--Fevereiro-2019.pdf> Acesso em 25 Fev. 2019.

Cabello, F. C. (2007). Salmon aquaculture and transmission of the fish tapeworm. Emerging Infectious Diseases, 13(1), 169. DOI: https://doi.org/10.3201/eid1301.060875

Cimerman, B., \& Cimerman, S. (2005). Parasitologia humana e seus fundamentos gerais. In Parasitologia humana e seus fundamentos gerais (p. 390). Atheneu Editora.

Emmel, V. E., Inamine, E., Secchi, C., Brodt, T. C. Z., Amaro, M. C. O., Cantarelli, V. V., \& Spalding, S. (2006). Diphyllobothrium latum: relato de caso no Brasil. Revista Da Sociedade Brasileira de Medicina Tropical, 39(1), 82-84. DOI: https://doi.org/10.1590/s0037-86822006000100017

FOOd and Agriculture Organization Of The United Natioans - FAO. (2018). The State of World Fisheries and Aquaculture 2018 - Meeting the sustainable development goals. Rome. Disponível em: http://www.fao.org/state-of-fisheries-aquaculture. Acesso em 10 fev 2019.

Kuchta, R., Esteban, J.-G., Brabec, J., \& Scholz, T. (2014). Misidentification of Diphyllobothrium species related to global fish trade, Europe. Emerging Infectious Diseases, 20(11), 1955-1957. DOI: https://doi.org/10.3201/eid2011.140996

López-López, I., Cofrades, S., Ruiz-Capillas, C., \& Jiménez-Colmenero, F. (2009). Design and nutritional properties of potential functional frankfurters based on lipid formulation, added seaweed and low salt content. Meat Science, 83(2), 255-262. DOI: https://doi.org/10.1016/j.meatsci.2009.05.014

Luque, J. L. (2004). Biologia, epidemiologia e controle de parasitos de peixes. Revista Brasileira de Parasitologia Veterinária, 13(Supl 1), 161-165.

Machado, J. M., \& Marques, S. M. T. (2015). Difilobotríase humana pelo consumo de peixe: revisão de literatura Human diphyllobothriasis caused by fish consumption: a literature review. PUBVET, 8 , 2806-2887. DOI: https://doi.org/10.22256/pubvet.v8n23.1815

Marval, F., Gottstein, B., Weber, M., \& Wicht, B. (2013). Imported diphyllobothriasis in Switzerland: molecular methods to define a clinical case of Diphyllobothrium infection as Diphyllobothrium dendriticum, August 2010. Eurosurveillance, 18(3), pii: 20355. DOI: https://doi.org/10.2807/ese.18.03.20355-en

Mega, J. D., Galdos-Cardenas, G., \& Gilman, R. H. (2013). Tapeworm Infections. In A. J. Alan J. Magill, D. R. Hill, T. Solomon, \& E. T. Ryan (Eds.), Hunter's tropical medicine and emerging infectious disease (pp. 895-902). Saunders, Elsevier. DOI: https://doi.org/10.1016/b978-1-4160$\underline{4390-4.00126-0}$

Neves, D. P. (2005). Parasitologia humana. Editora Atheneu.

OMS, MS. Organização Mundial da Saúde. Zoonoses. Disponível em: http://www.who.int/topics/zoonoses/en/. Acesso em 4 abr 2019. 2016

Park, S. C., Keum, B., Jeen, Y.-T., \& Chun, H. J. (2011). Diphyllobothrium latum accidentally detected by colonoscopy. Digestive and Liver Disease, 43(8), 664. DOI: 
https://doi.org/10.1016/j.dld.2011.02.013

Rosas, R., \& Weitzel, T. (2014). Diphyllobothrium latum. Revista Chilena de Infectología, 31(2), 211212. DOI: https://doi.org/10.1001/jama.1932.02730380085033

Santos, F. L. N., \& Faro, L. B. (2005). The first confirmed case of Diphyllobothrium latum in Brazil. Memórias Do Instituto Oswaldo Cruz, 100(6), 585-586. DOI: https://doi.org/10.1590/S0074$\underline{02762005000600013}$

Sartori, A. G. O., \& Amancio, R. D. (2012). Pescado: importância nutricional e consumo no Brasil. Segurança Alimentar e Nutricional, 19(2), 83-93. DOI: https://doi.org/10.20396/san.v19i2.8634613

Scholz, T., \& Kuchta, R. (2016). Fish-borne, zoonotic cestodes (Diphyllobothrium and relatives) in cold climates: a never-ending story of neglected and (re)-emergent parasites. Food and Waterborne Parasitology, 4, 23-38. DOI: https://doi.org/10.1016/j.fawpar.2016.07.002

Sonoda, D. Y., \& Shirota, R. (2012). Consumo de pescado no Brasil fica abaixo da média internacional. Visão Agrícola, 8(11), 145-147.

Tavares, G. C., Leibowitz, M. P., Leal, C. A. G., \& Figueiredo, H. C. P. (2018). Zoonoses emergentes associadas ao consumo e à manipulação de pescado. Cadernos Técnicos da Escola de Veterinária da UFMG, 89, 7-38.

United States Food and Drug Administration-FDA. (2011). Fish and Fishery Products Hazards and Controls Guidance. Fourth Edition, U.S. Department of Health and Human Services Food and Drug Administration Center for Food Safety and Applied Nutrition (240) 402-2300 April 2011.

World Health Organization - WHO. (1995). Model Prescribing Information: Drugs Used in Parasitic Diseases - 2nd ed. Geneva, World Health Organization.

\section{Histórico do artigo:}

Recebido: 24 de outubro, 2020

Aprovado: 3 de novembro, 2020.

Disponível online: 1 de março de 2021
Licenciamento: Este artigo é publicado na modalidade Acesso Aberto sob a licença Creative Commons Atribuição 4.0 (CC-BY 4.0), a qual permite uso irrestrito, distribuição, reprodução em qualquer meio, desde que o autor e a fonte sejam devidamente creditados. 